\title{
Anisotropic multi-phase-field model: Interfaces and junctions
}

\author{
B. Nestler ${ }^{1}$ and A. A. Wheeler ${ }^{2}$ \\ ${ }^{1}$ Foundry Institute, University of Aachen, Intzestrasse 5, D-52072 Aachen, Germany \\ ${ }^{2}$ Faculty of Mathematical Studies, University of Southampton, Southampton, United Kingdom
}

(Received 27 October 1997)

\begin{abstract}
In this paper we bring together and extend two recent developments in phase-field models, namely, a phase-field model of a multiphase system [I. Steinbach et al., Physica D 94, 135 (1996)] and the extension of the Cahn-Hoffman $\xi$-vector theory of anisotropic sharp interfaces to phase-field models [A. A. Wheeler and G. B. McFadden, Eur. J. Appl. Math. 7, 369 (1996); Proc. R. Soc. London, Ser. A 453, 1611 (1997)]. We develop the phase-field model of a multiphase system proposed by Steinbach et al. to include both surface energy and interfacial kinetic anisotropy. We show that this model may be compactly expressed in terms of generalized Cahn-Hoffman $\xi$ vectors. This generalized Cahn-Hoffman $\xi$-vector formalism is subsequently developed to include the notion of a stress tensor, which is used to succinctly derive the leading-order conditions at both moving interfaces and stationary multijunctions in the sharp interface limit. [S1063-651X(98)03703-9]
\end{abstract}

PACS number(s): 64.60.-i, 68.35.Rh, 81.10.Jt

\section{INTRODUCTION}

In this paper we present and analyze a phase-field model that describes multiphase systems and includes both anisotropic surface energies and mobilities. It provides a model of a wide variety of different situations such as eutectic and peritectic alloys as well as the motion of grain boundaries.

A phase-field model of a solid-liquid interface was proposed by Langer [1] and was subsequently developed by a number of workers [2-5]. Wheeler, Boettinger, and McFadden [6] provided a phase-field model of a simple isothermal binary alloy. Subsequently, several authors have extended their work to model more realistic nonisothermal situations $[7,8]$ as well as eutectic alloys $[9,10]$ and solute trapping [11-14]. Phase-field models of a pure material have also been extended to include anisotropy of the surface energy and interface mobility, by [2,15-17]. Recently, Wheeler and McFadden [18] have developed the notion of a generalized $\xi$ vector for phase-field models with anisotropic surface energy that extends the original idea of a $\xi$ vector that Cahn and Hoffman $[19,20]$ developed for sharp interface models. Subsequently, Wheeler and McFadden [21] extended this idea to formulate a conservation law in terms of a stress tensor related to the $\xi$ vector, which they used to investigate the force balance at a multijunction.

Several authors [22-28] have extended the phase-field methodology to develop models involving a vector-valued order parameter in an attempt to consider a wider range of phase transitions such as multicomponent or multiphase systems. In particular, Steinbach et al. [27] developed a phasefield model of a multiphase system in which a phase field is associated with each phase present. In this work an underlying free-energy functional was chosen that involved the pairwise interactions between all the different phases. The resulting governing equations were used to conduct numerical simulations of a variety of situations and demonstrated the feasibility of this approach, in particular in relation to the qualitative simulation of a number of important growth phenomena in peritectic and eutectic systems [29,30]. In related work Garcke, Nestler, and Stoth [26] used a formal asymptotic analysis to determine the asymptotic singular limit of a multiphase Allen-Cahn system.

The focus of this paper is to develop the model of Steinbach et al. to include the effect of anisotropic surface energies associated with the interfaces between the different phases, as well as to include a more general form of the anisotropic mobility of the interfaces. To this end we draw on the work of Wheeler and McFadden [18,21] to place the dimensionless form of the model in the setting of the generalized Cahn-Hoffman $\xi$ vector. This allows us to conduct succinctly the sharp interface asymptotics and to recover the correct form of the Gibbs-Thomson-Herring equation for an interface in three dimensions as well as the force balance at a multijunction between anisotropic interfaces. We show that the $\xi$-vector formalism is a powerful and elegant tool not only for investigating surface energy anisotropy in the context of a sharp interface theory, for which it was originally developed, but also for the complicated diffuse interface theory described here.

In the sharp interface theory in which an anisotropic surface energy $\gamma(\vec{n})$ is associated with an interface $S$, which has a unit normal $\vec{n}$, the $\xi$ vector is defined by

$$
\vec{\xi}=\vec{\nabla} \gamma(\vec{r})
$$

where $\gamma(\vec{r})$ is the homogeneous extension of degree one of $\gamma(\vec{n})$. In spherical polar coordinates $\vec{\xi}$ may be expressed as

$$
\vec{\xi}=\gamma(\theta, \phi) \vec{e}_{r}+\frac{\partial \gamma(\theta, \phi)}{\partial \theta} \vec{e}_{\theta}+\frac{1}{\sin \theta} \frac{\partial \gamma(\theta, \phi)}{\partial \phi} \vec{e}_{\phi}
$$

Cahn and Hoffman showed that the Gibbs-Thomson-Herring equation may be compactly expressed as

$$
T_{I}=T_{M}-\frac{1}{L} \vec{\nabla}_{S} \cdot \vec{\xi}
$$

where $T_{I}$ and $T_{M}$ are the interface and melting temperatures, respectively, and $L$ is the latent heat per unit volume. They were able to relate the $\xi$ vector to the stress in the interface 
and showed that at a multijunction, comprising the confluence on $m$ interfaces, the equilibrium force balance could be expressed as

$$
\vec{l} \times \sum_{k=1}^{m} \vec{\xi}_{k}=\overrightarrow{0}
$$

where $\vec{l}$ is the unit vector parallel to the junction and $\vec{\xi}_{k}, k$ $=1, \ldots, m$, are the $\xi$ vectors associated with each interface.

In Sec. II we develop our anisotropic phase-field model, derive the governing equations, and express the model in dimensionless form. In Sec. III we show how generalized $\xi$ vectors may be associated with this model. In Sec. IV we exploit this formalism to investigate the sharp interface limit of interfaces and derive the Gibbs-Thomson-Herring equation. In Sec. V we develop a stress tensor for our model and use it to obtain, in a compact way, the force balance at an equilibrium multijunction.

\section{MODEL}

We consider an isothermal system in which $N$ different bulk phases may exist. Following the approach of Steinbach et al. [27], we introduce $N$ corresponding phase-field variables $\phi_{1}, \ldots, \phi_{N}$ with $0 \leqslant \phi_{\alpha} \leqslant 1$ for $\alpha=1, \ldots, N$. We assume that the system can reside exclusively in bulk phase $n$ $(1 \leqslant n \leqslant N)$ at a point in space if $\phi_{n}=1$ and $\phi_{\alpha}=0$ for all $\alpha \neq n, 1 \leqslant \alpha \leqslant N$. We therefore impose the constraint that

$$
\sum_{\alpha=1}^{N} \phi_{\alpha}=1
$$

The free-energy functional associated with the system is given by

$$
\mathcal{F}=\int_{V} \mathcal{L}(\vec{\phi}, \vec{\nabla} \vec{\phi}) d V
$$

where the Lagrangian energy density $\mathcal{L}(\vec{\phi}, \vec{\nabla} \vec{\phi})$ depends on the vector $\vec{\phi}=\left(\vec{\phi}_{1}, \ldots, \phi_{N}\right)$ and its gradients $\vec{\nabla} \vec{\phi}$ $=\left(\vec{\nabla} \phi_{1}, \ldots, \vec{\nabla} \phi_{N}\right)$.

In the case when all the interfaces between the different bulk phases possess isotropic surface energy $\mathcal{L}(\vec{\phi}, \vec{\nabla} \vec{\phi})$ can be defined by (see Ref. [27])

$$
\begin{aligned}
\mathcal{L}^{\text {iso }}(\vec{\phi}, \vec{\nabla} \vec{\phi})= & \sum_{\beta=1}^{N} \sum_{\alpha=1}^{\beta}\left[\frac{1}{2} \eta_{\alpha \beta}^{2}\left|\vec{r}_{\alpha \beta}\right|^{2}+\widetilde{g}_{\alpha \beta}(\vec{\phi})\right] \\
& +\sum_{\alpha=1}^{N} \widetilde{h}_{\alpha}(\vec{\phi}, T),
\end{aligned}
$$

where $\eta_{\alpha \beta}$ are constant gradient energy coefficients and the vector $\vec{r}_{\alpha \beta}$ appearing in the gradient energy terms is given by

$$
\vec{r}_{\alpha \beta}=\phi_{\alpha} \vec{\nabla} \phi_{\beta}-\phi_{\beta} \vec{\nabla} \phi_{\alpha} .
$$

In this model the possible $N ! /[(N-2) ! 2 !]$ interactions between the $N$ bulk phases are described by a sum over the pairwise intercorrelation energies of the different phase fields denoted by $\tilde{g}_{\alpha \beta}(\vec{\phi})$. Using physical arguments Steinbach et al. [27] assumed that the movement of the two phase boundaries dominates the adjustment of triple or multiple junctions, which possess a negligible dynamics of their own. Hence energy terms that depend on more than two phases are neglected. The pairwise intercorrelation energy terms are specified to be double-well potentials of the form

$$
\tilde{g}_{\alpha \beta}(\vec{\phi})=\frac{1}{4 a_{\alpha \beta}} g_{\alpha \beta}(\vec{\phi})=\frac{1}{4 a_{\alpha \beta}} \phi_{\alpha}^{2} \phi_{\beta}^{2} .
$$

The parameter $1 / a_{\alpha \beta}$ is proportional to the pairwise barrier height of the double well. The terms $\widetilde{h}_{\alpha}(\vec{\phi}, T)$ describe the free-energy density of each bulk phase and are assumed to be given by

$$
\widetilde{h}_{\alpha}(\vec{\phi}, T)=\widetilde{b}_{\alpha}(T) \phi_{\alpha} .
$$

The parameters $\eta_{\alpha \beta}$ and $1 / a_{\alpha \beta}$ are defined for each interface between bulk phases labeled $\alpha$ and $\beta$. They may be related to measurable, physical quantities (see Ref. [18]) such as the interface thickness $\ell_{\alpha \beta}$ and surface energy $\gamma_{\alpha \beta}$ through the identities

$$
\ell_{\alpha \beta}=2 \sqrt{2} \eta_{\alpha \beta} \sqrt{a_{\alpha \beta}}, \quad \gamma_{\alpha \beta}=\frac{\ell_{\alpha \beta}}{24 a_{\alpha \beta}} .
$$

From the obvious symmetry requirements that $\ell_{\alpha \beta}=\ell_{\beta \alpha}$ and $\gamma_{\alpha \beta}=\gamma_{\beta \alpha}$ we therefore deduce that

$$
\eta_{\alpha \beta}=\eta_{\beta \alpha}, \quad a_{\alpha \beta}=a_{\beta \alpha} .
$$

The form of the free-energy functional and the symmetry properties of the parameters ensure the reproduction of the standard isotropic phase-field model in the case of only two phases in the system, e.g., a liquid and a solid phase [2].

In an analogous way to the anisotropic phase-field model described by Wheeler and McFadden [18] for a solid-liquid interface, we extend the isotropic Lagrangian density (7) to the anisotropic situation by writing

$$
\begin{aligned}
\mathcal{L}(\vec{\phi}, \vec{\nabla} \vec{\phi})= & \sum_{\beta=1}^{N} \sum_{\alpha=1}^{\beta}\left\{\frac{1}{2} \eta_{\alpha \beta}^{2}\left[\Gamma_{\alpha \beta}\left(\vec{r}_{\alpha \beta}\right)\right]^{2}+\widetilde{g}_{\alpha \beta}(\vec{\phi})\right\} \\
& +\sum_{\alpha=1}^{N} \widetilde{b}_{\alpha}(T) \phi_{\alpha}+\widetilde{\lambda}\left[\sum_{\alpha=1}^{N} \phi_{\alpha}-1\right],
\end{aligned}
$$

where we have invoked the constraint (5) with a Lagrange multiplier $\widetilde{\lambda}$. Here $\Gamma_{\alpha \beta}\left(\vec{r}_{\alpha \beta}\right)$ is defined to be a homogeneous degree-one function of its argument. It imparts anisotropic surface energy to the interfaces. When $\Gamma_{\alpha \beta}\left(\vec{r}_{\alpha \beta}\right)=\left|\vec{r}_{\alpha \beta}\right|$ the isotropic Lagrangian is recovered; otherwise we anticipate that the surface energy of the interfaces is given by

$$
\gamma_{\alpha \beta}\left(\vec{n}_{\alpha \beta}\right)=\frac{\eta_{\alpha \beta} \sqrt{a_{\alpha \beta}}}{6 \sqrt{2} a_{\alpha \beta}} \Gamma_{\alpha \beta}\left(\vec{n}_{\alpha \beta}\right),
$$




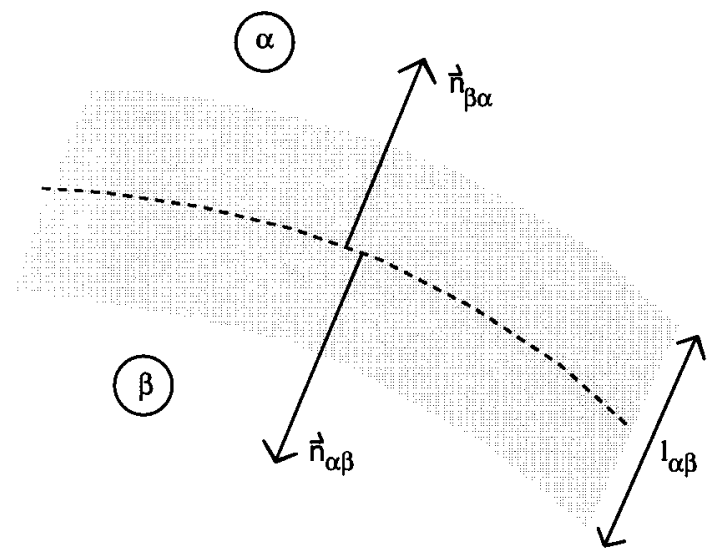

FIG. 1. Schematic diagram of a diffuse interface of thickness $\ell_{\alpha \beta}$ between two bulk phases labeled $\alpha$ and $\beta$. The orientation of the two unit normal vectors $\vec{n}_{\alpha \beta}$ and $\vec{n}_{\beta \alpha}$ is shown.

where $\vec{n}_{\alpha \beta}$ is the unit normal to the interface between phases labeled $\alpha$ and $\beta$; see Fig. 1. We note that $\gamma_{\alpha \beta}$ is a homogeneous degree-one function of its argument, which it inherits from $\Gamma_{\alpha \beta}$.

To proceed we nondimensionalize the free-energy density (and $\widetilde{\lambda}$ ) with respect to $b_{1}$, length with respect to $R$, which represents a typical radius of curvature of the interfaces, and the surface energy $\gamma_{\alpha \beta}$ with respect to $b_{1} R$. Using Eqs. (11) and (12), the dimensionless Lagrangian density may be expressed as

$$
\begin{aligned}
\mathcal{L}(\vec{\phi}, \vec{\nabla} \vec{\phi})= & \sum_{\beta=1}^{N} \sum_{\alpha=1}^{\beta}\left[36 \epsilon_{\alpha \beta} \gamma_{\alpha \beta}^{2}\left(\vec{r}_{\alpha \beta}\right)+\frac{1}{4 \epsilon_{\alpha \beta}} g_{\alpha \beta}(\vec{\phi})\right] \\
& +\sum_{\alpha=1}^{N} b_{\alpha}(T) \phi_{\alpha}+\lambda\left[\sum_{\alpha=1}^{N} \phi_{\alpha}-1\right]
\end{aligned}
$$

where $\epsilon_{\alpha \beta}=a_{\alpha \beta} b_{1}, b_{\alpha}=\widetilde{b}_{\alpha} / b_{1}, \lambda=\widetilde{\lambda} / b_{1}$, and we note that

$$
\epsilon_{\alpha \beta}=\epsilon_{\beta \alpha} .
$$

The governing equations are given by the gradient flow

$$
\frac{\partial \phi_{\mu}}{\partial t}=-M(\vec{\nabla} \vec{\phi}) \frac{\delta \mathcal{F}}{\delta \phi_{\mu}} \quad \text { for } \mu=1, \ldots, N
$$

where $M(\vec{\nabla} \vec{\phi})$ represents a dimensionless anisotropic mobility. These equations may be recast in terms of the Lagrangian density as

$$
\frac{\partial \phi_{\mu}}{\partial t}=M(\vec{\nabla} \vec{\phi})\left\{\vec{\nabla}\left(\frac{\partial \mathcal{L}}{\partial \nabla \phi_{\mu}}\right)-\frac{\partial \mathcal{L}}{\partial \phi_{\mu}}\right\}
$$

It is convenient to choose the mobility to be of the form

$$
M(\vec{\nabla} \vec{\phi})=\frac{\left\{\sum_{\beta=1}^{N} \sum_{\alpha=1}^{N} \phi_{\alpha} \mu_{\alpha \beta}\left(\vec{r}_{\alpha \beta}\right)\right\}}{\left\{72 \sum_{\beta=1}^{N} \sum_{\alpha=1}^{N} \epsilon_{\alpha \beta} \phi_{\alpha} \gamma_{\alpha \beta}\left(\vec{r}_{\alpha \beta}\right)\right\}},
$$

where $\mu_{\alpha \beta}\left(\vec{r}_{\alpha \beta}\right)$ are homogeneous degree-one functions such that $\mu_{\alpha \beta}\left(\vec{r}_{\alpha \beta}\right)=\mu_{\beta \alpha}\left(\vec{r}_{\beta \alpha}\right)$. As shown below, the function $\mu_{\alpha \beta}\left(\vec{n}_{\alpha \beta}\right)$ may be interpreted, in the sharp interface limit, as the dimensionless anisotropic kinetic mobility of the interface between the bulk phases labeled $\alpha$ and $\beta$.

\section{THE $\xi$-VECTOR FORMULATION}

Our next aim is to define the $\xi$ vector for the multi-phasefield model described above. Consider the interfacial region between two bulk phases labeled $\alpha$ and $\beta$. We will assume that in this region $\phi_{\alpha}$ and $\phi_{\beta}$ are the only phase fields that are nonzero, so that $\phi_{\alpha}+\phi_{\beta}=1$. We denote the surface in this interfacial region defined by $\phi_{\alpha}=\phi_{\beta}=\frac{1}{2}$ as $S_{\alpha \beta}$. The unit vectors to this surface are denoted by $\vec{n}_{\alpha \beta}$ and $\vec{n}_{\beta \alpha}$; see Fig. 1 for their orientation. As we show in Sec. IV, the only two nontrivial governing equations, for $\phi_{\alpha}$ and $\phi_{\beta}$, are both similar to the phase-field equation discussed by Wheeler and McFadden [18]. Hence, in the sharp interface limit $\epsilon_{\alpha \beta}$ $=\epsilon_{\beta \alpha} \rightarrow 0$, the surface energy of the interface associated with $S_{\alpha \beta}$ is described, to leading order, by $\gamma_{\alpha \beta}\left(\vec{n}_{\alpha \beta}\right)$ or $\gamma_{\beta \alpha}\left(\vec{n}_{\beta \alpha}\right)$. Because $\vec{n}_{\alpha \beta}=-\vec{n}_{\beta \alpha}$, we obtain the symmetry condition

$$
\gamma_{\alpha \beta}\left(\vec{n}_{\alpha \beta}\right)=\gamma_{\beta \alpha}\left(-\vec{n}_{\alpha \beta}\right) .
$$

The functions $\gamma_{\alpha \beta}\left(\vec{r}_{\alpha \beta}\right)$ have been extended as degree-one homogeneous functions of their arguments [see Eq. (14)], so that

$$
\gamma_{\alpha \beta}\left(\zeta \vec{r}_{\alpha \beta}\right)=\zeta \gamma_{\alpha \beta}\left(\vec{r}_{\alpha \beta}\right) \text { for all } \zeta \in \mathbb{R} \text {, }
$$

and hence it follows from Eqs. (20) and (21) that

$$
\gamma_{\alpha \beta}\left(\vec{r}_{\alpha \beta}\right)=\gamma_{\beta \alpha}\left(\vec{r}_{\beta \alpha}\right) .
$$

In the spirit of Wheeler and McFadden [18], we define the set of $\xi$ vectors $\vec{\xi}_{\alpha \beta}\left(\vec{r}_{\alpha \beta}\right)$ by

$$
\vec{\xi}_{\alpha \beta}\left(\vec{r}_{\alpha \beta}\right)=\frac{\partial \gamma_{\alpha \beta}\left(\vec{r}_{\alpha \beta}\right)}{\partial \vec{r}_{\alpha \beta}}=\vec{\nabla}_{\vec{r}_{\alpha \beta}} \gamma_{\alpha \beta}\left(\vec{r}_{\alpha \beta}\right),
$$

which are consequently homogeneous functions of degree zero. From Eqs. (22) and (23) it follows that

$$
\vec{\xi}_{\alpha \beta}=-\vec{\xi}_{\beta \alpha} .
$$

We note from the definition of $\vec{\xi}_{\alpha \beta}\left(\vec{r}_{\alpha \beta}\right)$ that

$$
\gamma_{\alpha \beta}\left(\vec{r}_{\alpha \beta}\right)=\vec{r}_{\alpha \beta} \cdot \vec{\xi}_{\alpha \beta},
$$


which follows from the calculus of degree-one homogenous functions.

We observe that

$$
\begin{aligned}
\frac{\partial \mathcal{L}}{\partial \vec{\nabla} \phi_{\mu}}= & 72\left\{\sum_{\alpha<\mu} \epsilon_{\alpha \mu} \gamma_{\alpha \mu}\left(\vec{r}_{\alpha \mu}\right) \frac{\partial \gamma_{\alpha \mu}}{\partial \vec{\nabla} \phi_{\mu}}\right. \\
& \left.+\sum_{\beta>\mu} \epsilon_{\mu \beta} \gamma_{\mu \beta}\left(\vec{r}_{\mu \beta}\right) \frac{\partial \gamma_{\mu \beta}\left(\vec{r}_{\mu \beta}\right)}{\partial \vec{\nabla} \phi_{\mu}}\right\} \\
= & 72\left\{\sum_{\alpha<\mu} \epsilon_{\alpha \mu} \gamma_{\alpha \mu}\left(\vec{r}_{\alpha \mu}\right) \frac{\partial \gamma_{\alpha \mu}}{\partial \vec{r}_{\alpha \mu}} \frac{\partial \vec{r}_{\alpha \mu}}{\partial \vec{\nabla} \phi_{\mu}}\right. \\
& \left.+\sum_{\beta>\mu} \epsilon_{\mu \beta} \gamma_{\mu \beta}\left(\vec{r}_{\mu \beta}\right) \frac{\partial \gamma_{\mu \beta}}{\partial \vec{r}_{\mu \beta}} \frac{\partial \vec{r}}{\partial \beta} \vec{\nabla}_{\mu}\right\} \\
= & 72\left\{\sum_{\alpha \neq \mu}^{N} \epsilon_{\alpha \mu} \gamma_{\alpha \mu}\left(\vec{r}_{\alpha \mu}\right) \vec{\xi}_{\alpha \mu} \phi_{\alpha}\right\}
\end{aligned}
$$

where we have used the symmetry relations for Eqs. (16), (22), and (24). A similar calculation gives that

$$
\begin{aligned}
\frac{\partial \mathcal{L}}{\partial \phi_{\mu}}= & \sum_{\alpha \neq \mu}^{N}\left[-72 \epsilon_{\alpha \mu} \gamma_{\alpha \mu}\left(\vec{r}_{\alpha \mu}\right) \vec{\xi}_{\alpha \mu} \cdot \vec{\nabla} \phi_{\alpha}+\frac{1}{4 \epsilon_{\alpha \mu}} \frac{\partial g_{\alpha \mu}}{\partial \phi_{\mu}}\right] \\
& +b_{\mu}(T)+\lambda .
\end{aligned}
$$

Hence the governing equations (18) may be written as

$$
\begin{aligned}
\frac{1}{M(\vec{\nabla} \vec{\phi})} \frac{\partial \phi_{\mu}}{\partial t}= & \sum_{\alpha \neq \mu}^{N}\left[7 2 \epsilon _ { \alpha \mu } \left\{\vec{\nabla} \cdot\left[\gamma_{\alpha \mu}\left(\vec{r}_{\alpha \mu}\right) \vec{\xi}_{\alpha \mu} \phi_{\alpha}\right]\right.\right. \\
& \left.\left.+\gamma_{\alpha \mu}\left(\vec{r}_{\alpha \mu}\right) \vec{\xi}_{\alpha \mu} \cdot \vec{\nabla} \phi_{\alpha}\right\}-\frac{1}{4 \epsilon_{\alpha \mu}} \frac{\partial g_{\alpha \mu}}{\partial \phi_{\mu}}\right] \\
& -\beta_{\mu}(T)-\lambda
\end{aligned}
$$

for $\mu=1, \ldots, N$. The Lagrange multiplier may be found, using the constraint (5), to be

$$
\begin{aligned}
\lambda= & \sum_{\mu=1}^{N} \phi_{\mu}\left\{\sum _ { \alpha \neq \mu } ^ { N } \left[7 2 \epsilon _ { \alpha \mu } \left\{\vec{\nabla} \cdot\left[\gamma_{\alpha \mu}\left(\vec{r}_{\alpha \mu}\right) \vec{\xi}_{\alpha \mu} \phi_{\alpha}\right]\right.\right.\right. \\
& \left.\left.+\gamma_{\alpha \mu}\left(\vec{r}_{\alpha \mu}\right) \vec{\xi}_{\alpha \mu} \cdot \vec{\nabla} \phi_{\alpha}\right\}-\frac{1}{4 \epsilon_{\alpha \mu}} \frac{\partial g_{\alpha \mu}}{\partial \phi_{\mu}}\right] \\
& \left.-\frac{1}{M(\vec{\nabla} \vec{\phi})} \frac{\partial \phi_{\mu}}{\partial t}-b_{\mu}(T)\right\} .
\end{aligned}
$$

\section{INTERFACES}

We now briefly consider a curved interface between two bulk phases labeled $\alpha$ and $\beta$ in the sharp interface limit $\epsilon_{\alpha \beta}=\epsilon_{\beta \alpha} \rightarrow 0$. Using the symmetry conditions for $\mu_{\alpha \beta}, \epsilon_{\alpha \beta}, \gamma_{\alpha \beta}$ and the constraint $\phi_{\alpha}+\phi_{\beta}=1$ the two nontrivial governing equations are

$$
\begin{gathered}
72 \epsilon_{\beta \alpha}\left\{\vec{\nabla} \cdot\left[\gamma_{\beta \alpha}\left(\vec{r}_{\beta \alpha}\right) \vec{\xi}_{\beta \alpha} \phi_{\beta}\right]+\gamma_{\beta \alpha} \vec{\xi}_{\beta \alpha} \cdot \vec{\nabla} \phi_{\beta}\right\}-\frac{1}{4 \epsilon_{\beta \alpha}} \frac{\partial g_{\beta \alpha}}{\partial \phi_{\alpha}} \\
-b_{\alpha}-\lambda=72 \epsilon_{\beta \alpha} \frac{\gamma_{\beta \alpha}\left(\vec{r}_{\beta \alpha}\right)}{\mu_{\beta \alpha}\left(\vec{r}_{\beta \alpha}\right)} \frac{\partial \phi_{\alpha}}{\partial t}, \\
72 \epsilon_{\alpha \beta}\left\{\vec{\nabla} \cdot\left[\gamma_{\alpha \beta}\left(\vec{r}_{\alpha \beta}\right) \vec{\xi}_{\alpha \beta} \phi_{\alpha}\right]+\gamma_{\alpha \beta} \vec{\xi}_{\alpha \beta} \cdot \vec{\nabla} \phi_{\alpha}\right\}-\frac{1}{4 \epsilon_{\alpha \beta}} \frac{\partial g_{\alpha \beta}}{\partial \phi_{\beta}} \\
-b_{\beta}-\lambda=72 \epsilon_{\alpha \beta} \frac{\gamma_{\alpha \beta}\left(\vec{r}_{\alpha \beta}\right)}{\mu_{\alpha \beta}\left(\vec{r}_{\alpha \beta}\right)} \frac{\partial \phi_{\beta}}{\partial t} .
\end{gathered}
$$

We subtract these two equations and use the additional symmetry condition for $\vec{\xi}_{\alpha \beta}$ to obtain

$$
\begin{gathered}
72 \epsilon_{\beta \alpha} \vec{\nabla} \cdot\left[\gamma_{\beta \alpha}\left(\vec{\nabla} \phi_{\alpha}\right) \vec{\xi}_{\beta \alpha}\right]-\frac{1}{\epsilon_{\beta \alpha}} \phi_{\alpha}\left(1-\phi_{\alpha}\right)\left(\frac{1}{2}-\phi_{\alpha}\right) \\
-\Delta b_{\beta \alpha}=144 \epsilon_{\beta \alpha} \frac{\gamma_{\beta \alpha}\left(\vec{\nabla} \phi_{\alpha}\right)}{\mu_{\beta \alpha}\left(\vec{\nabla} \phi_{\alpha}\right)} \frac{\partial \phi_{\alpha}}{\partial t}
\end{gathered}
$$

where $\Delta b_{\alpha \beta}=b_{\beta}-b_{\alpha}$ is the dimensionless bulk free-energy difference between the two phases and is related to their mutual latent heat $L_{\alpha \beta}$ by

$$
\Delta b_{\alpha \beta}=\frac{L_{\alpha \beta}}{b_{1}} \frac{T-T_{\alpha \beta}^{M}}{T_{\alpha \beta}^{M}},
$$

where $T_{\alpha \beta}^{M}$ is their melting point, provided the temperature $T$ is close to $T_{\alpha \beta}^{M}$. A similar equation holds for $\phi_{\beta}$.

This is the same form of the phase-field equation considered by Wheeler and McFadden [21], who studied its sharp interface limit. We briefly outline their results and interpret them in the context of the multi-phase-field model. We introduce a body-fitted coordinate system in which $r$ measures the distance from the surface $S_{\alpha \beta}$ and increases towards the bulk phase $\beta$. To examine the solution in the interface region we rescale $r$ to the interface thickness, which is $O\left(\epsilon_{\alpha \beta}\right)$, and therefore write $r=\epsilon_{\alpha \beta} \rho$, where $\rho=O(1)$. The leading-order solution for $\phi_{\alpha}$, denoted by $\phi_{\alpha}^{(0)}(\rho)$, satisfies

$$
72\left[\gamma_{\alpha \beta}\left(\vec{n}_{\alpha \beta}\right)\right]^{2} \frac{d^{2} \phi_{\alpha}^{(0)}}{d \rho^{2}}-\phi_{\alpha}^{(0)}\left(1-\phi_{\alpha}^{(0)}\right)\left(\frac{1}{2}-\phi_{\alpha}^{(0)}\right)=0
$$

Hence

$$
\phi_{\alpha}^{(0)}=\frac{1}{2}\left[1-\tanh \left(\frac{\rho}{24 \gamma_{\alpha \beta}\left(\vec{n}_{\alpha \beta}\right)}\right)\right]
$$

and we note that the leading-order approximation for $\phi_{\alpha}$ in the interfacial layer satisfies the identity 


$$
\int_{-\infty}^{+\infty}\left[\frac{d \phi_{\alpha}^{(0)}}{d \rho}\right]^{2} d \rho=\frac{1}{72 \gamma_{\alpha \beta}\left(\vec{n}_{\alpha \beta}\right)}
$$

The first integral of Eq. (34) is

$$
36\left[\gamma_{\alpha \beta}\left(\vec{n}_{\alpha \beta}\right)\right]^{2}\left[\frac{d \phi_{\alpha}^{(0)}}{d \rho}\right]^{2}-\frac{1}{4}\left[\phi_{\alpha}^{(0)}\right]^{2}\left(1-\phi_{\alpha}^{(0)}\right)^{2}=0
$$

and hence the leading-order approximation to the Lagrangian density is

$$
\mathcal{L}(\vec{\phi}, \vec{\nabla} \vec{\phi})=\frac{72}{\epsilon_{\alpha \beta}}\left[\gamma_{\alpha \beta}\left(\vec{n}_{\alpha \beta}\right)\right]^{2}\left[\frac{d \phi_{\alpha}^{(0)}}{d \rho}\right]^{2}
$$

Wheeler and McFadden went on to consider the next-order problem. The solvability condition that arises gives

$$
\Delta b_{\alpha \beta}=-\vec{\nabla}_{S_{\alpha \beta}} \cdot \vec{\xi}_{\alpha \beta}-v_{n_{\alpha \beta}} / \mu_{\alpha \beta}\left(\vec{n}_{\alpha \beta}\right)
$$

on the sharp interface, given by $S_{\alpha \beta}$, between the two phases, where $v_{n_{\alpha \beta}}$ is the normal interface velocity (measured positive when the bulk phase $\alpha$ grows). The term $\mu_{\alpha \beta}\left(\vec{n}_{\alpha \beta}\right)$ clearly plays the role of a dimensionless kinetic mobility. This is the dimensionless form of the GibbsThomson-Herring equation. For this interface $\vec{\xi}_{\alpha \beta}$ plays the role of the Cahn-Hoffman $\xi$ vector.

\section{JUNCTIONS}

We now exploit the $\xi$-vector formalism to investigate stationary multijunctions where $m(\leqslant N)$ bulk phases meet, to show that the classical force balance holds in the sharp interface limit. To this end we first show how a stress tensor may be developed for the multi-phase-field model. The stationary form of the governing equations provides the EulerLagrange equations for the minimization of the total free energy $\mathcal{F}$. Because the Lagrangian density is independent of the spatial coordinates we may appeal to Noether's theorem [31], which states that therefore there exists a conservation law given by

$$
\vec{\nabla} \cdot \Xi=0
$$

where

$$
\Xi=\sum_{\mu=1}^{N} \vec{\nabla} \phi_{\mu} \otimes \frac{\partial \mathcal{L}}{\partial \vec{\nabla} \phi_{\mu}}-\mathcal{L} I
$$

By Eq. (26) this tensor may be written as

$$
\Xi=72 \sum_{\mu=1}^{N} \sum_{\alpha \neq \mu}^{N} \epsilon_{\alpha \mu} \gamma_{\alpha \mu}\left(\vec{r}_{\alpha \mu}\right) \phi_{\alpha} \vec{\nabla} \phi_{\mu} \otimes \vec{\xi}_{\alpha \mu}-\mathcal{L} I .
$$

On applying the symmetry conditions (22) and (24), it may be more compactly expressed as

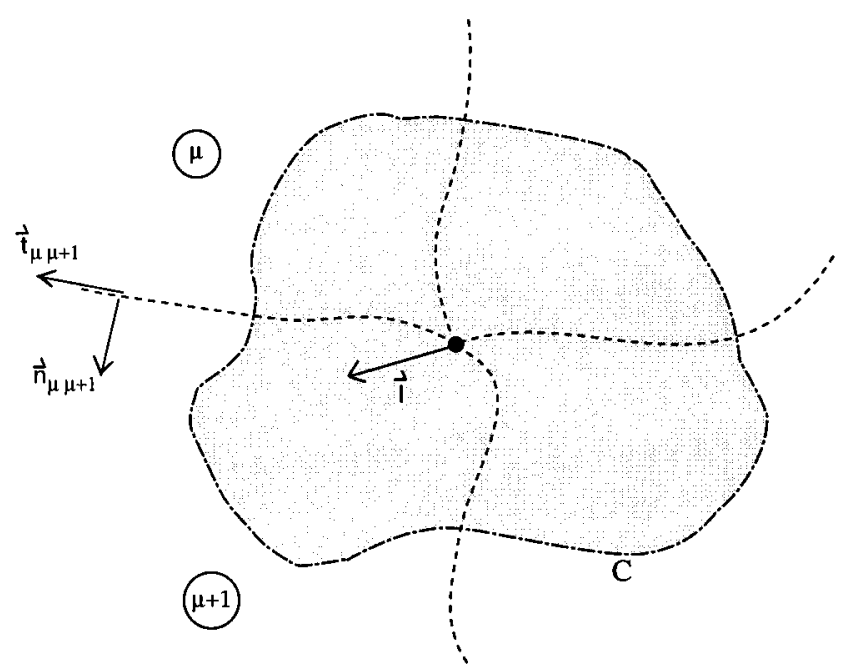

FIG. 2. Schematic diagram of a junction within a closed curve $C$ (for the particular case of four bulk phases) indicating the orientation of the unit tangential and normal vectors associated with each interface as well as the unit vector $\vec{l}$ that is parallel to the junction and is directed perpendicular to the plane of the diagram.

$$
\Xi=72 \sum_{\alpha<\beta} \epsilon_{\alpha \beta} \gamma_{\alpha \beta}\left(\vec{r}_{\alpha \beta}\right) \vec{r}_{\alpha \beta} \otimes \vec{\xi}_{\alpha \beta}-\mathcal{L} I .
$$

As discussed by Wheeler and McFadden [21] and in more detail by Anderson, McFadden, and Wheeler [32], 泟 may be shown to form the reversible part of the stress tensor in a more complete theory that entails deformation of matter.

We consider a multijunction that lies parallel to the unit vector $\vec{l}$ and hence we restrict our discussion to the plane with normal $\vec{l}$, in which case the surfaces $S_{\alpha \beta}$ may be regarded as curves in this plane. We assume that $m(\leqslant N)$ bulk phases meet at the multijunction and they are labeled in a counterclockwise fashion about the multijunction by $\alpha$ $=0,1,2, \ldots, m-1$ with corresponding phase fields $\phi_{0}, \phi_{1}, \phi_{2}, \ldots, \phi_{m-1}$. Because the junction is in equilibrium the bulk phases have equal bulk free-energy densities, which, without loss of generality, we may choose to be zero so that $b_{\mu}(T)=0$ for $\mu=0, \ldots, m-1$. Let $C$ be a closed curve such that the multijunction lies within $C$ and, for convenience, choose $C$ so that it perpendicularly intersects each of the phase boundaries emanating from the multijunction; see Fig. 2.

For clarity of exposition we assume all the constants $\epsilon_{\alpha \beta}$ are equal. (The more general case in which all the $\epsilon_{\alpha \beta}$ are different but proportional to a small parameter $\epsilon$ may be done in a similar way to the simpler case discussed in detail here.) We therefore write $\epsilon_{\alpha \beta}=\epsilon$ and consider the multijunction in the sharp interface limit $\epsilon \rightarrow 0$, in which case all the interfaces are of thickness $O(\epsilon)$. Let $\vec{n}_{\mu \mu+1}$ denote the unit normal of the curve $S_{\mu \mu+1}$ between the phases represented by the phase fields $\phi_{\mu}$ and $\phi_{\mu+1}(\bmod (m))$, where $0 \leqslant \mu$ $\leqslant m-1$. Thus the unit vector $\vec{t}_{\mu \mu+1}$ is the tangent vector to the curve $S_{\mu \mu+1}$ and is given by $\vec{t}_{\mu \mu+1}=\vec{n}_{\mu \mu+1} \times \vec{l}$. By construction $\vec{t}_{\mu \mu+1}$ lies in the direction normal to $C$. Since the 
stress tensor $\Xi$ is divergence-free the $\xi$-vector force balance for a multijunction follows from applying the divergence theorem

$$
\int_{C} \Xi \cdot \vec{\nu} d s=0
$$

where $\vec{\nu}$ is the outward unit normal to $C$. Because the bulk free-energy density of each phase is zero the only nonzero contributions to this integral arise from the parts of $C$ that intersect the interfacial regions. Hence, from Eq. (44), we obtain the force balance

$$
\sum_{\mu=0}^{m-1} \vec{F}_{\mu}=\overrightarrow{0}
$$

where $\vec{F}_{\mu}$ is the total force per unit length acting on the plane normal to the curve $S_{\mu \mu+1}$ in the direction of $\vec{t}_{\mu \mu+1}$. We now take the sharp interface limit $\epsilon \rightarrow 0$ and evaluate $\vec{F}_{\mu}$ to leading order. Integrating through the layer, $\vec{F}_{\mu}$ becomes

$$
\begin{aligned}
\vec{F}_{\mu} & =\epsilon \int_{-\infty}^{+\infty} \Xi \cdot \vec{t}_{\mu \mu+1} d \rho=\epsilon \int_{-\infty}^{+\infty}\left[72 \epsilon \gamma_{\mu \mu+1}\left(\vec{r}_{\mu \mu+1}\right) \vec{r}_{\mu \mu+1} \otimes \vec{\xi}_{\mu \mu+1}\left(\vec{r}_{\mu \mu+1}\right)-\mathcal{L} I\right] \cdot \vec{t}_{\mu \mu+1} d \rho \\
& =\epsilon \int_{-\infty}^{+\infty}\left\{72 \epsilon \gamma_{\mu \mu+1}\left(\vec{r}_{\mu \mu+1}\right)\left[\vec{\xi}_{\mu \mu+1}\left(\vec{r}_{\mu \mu+1}\right) \cdot \vec{t}_{\mu \mu+1}\right] \vec{r}_{\mu \mu+1}-\mathcal{L} \vec{t}_{\mu \mu+1}\right\} d \rho,
\end{aligned}
$$

where $\epsilon \rho$ denotes the distance from the interface from $S_{\mu \mu+1}$ in the direction of the unit normal vector $\vec{n}_{\mu \mu+1}$, i.e., through the interface. The leading-order approximations to the phase fields $\phi_{\mu}, \phi_{\mu+1}$ in the interfacial layer are given by Eq. (34). Thus we find that the leading-order approximations to $\vec{r}_{\mu \mu+1}$ and $\gamma_{\mu \mu+1}\left(\vec{r}_{\mu \mu+1}\right)$ are

$$
\begin{aligned}
\vec{r}_{\mu \mu+1} & \sim \frac{1}{\epsilon} \frac{\partial \phi_{\mu+1}^{(0)}}{\partial \rho} \vec{n}_{\mu \mu+1}, \\
\gamma_{\mu \mu+1}\left(\vec{r}_{\mu \mu+1}\right) & \sim \frac{1}{\epsilon} \frac{\partial \phi_{\mu+1}^{(0)}}{\partial \rho} \gamma_{\mu \mu+1}\left(\vec{n}_{\mu \mu+1}\right),
\end{aligned}
$$

where $r=\epsilon \rho$. Because the $\xi$ vector is a zero-degree homogeneous function, we note that

$$
\vec{\xi}_{\mu \mu+1}\left(\vec{r}_{\mu \mu+1}\right)=\vec{\xi}_{\mu \mu+1}\left(\vec{n}_{\mu \mu+1}\right)
$$

From Eq. (25) we find that

$$
\gamma_{\mu \mu+1}\left(\vec{n}_{\mu \mu+1}\right)=\vec{n}_{\mu \mu+1} \cdot \vec{\xi}_{\mu \mu+1}\left(\vec{n}_{\mu \mu+1}\right) .
$$

Applying these considerations, Eqs. (46) -(48), and using the leading-order form for the Lagrangian (38), we find, to leading order, that

$$
\begin{aligned}
\vec{F}_{\mu} & \sim \int_{-\infty}^{+\infty} 72 \gamma_{\mu \mu+1}\left(\vec{n}_{\mu \mu+1}\right)\left[\frac{\partial \phi_{\mu+1}^{(0)}}{\partial \rho}\right]^{2}\left[\left(\vec{\xi}_{\mu \mu+1} \cdot \vec{t}_{\mu \mu+1}\right) \vec{n}_{\mu \mu+1}-\gamma_{\mu \mu+1}\left(\vec{n}_{\mu \mu+1}\right) \vec{t}_{\mu \mu+1}\right] d \rho \\
& =\int_{-\infty}^{+\infty} 72 \gamma_{\mu \mu+1}\left(\vec{n}_{\mu \mu+1}\right)\left[\frac{\partial \phi_{\mu+1}^{(0)}}{\partial \rho}\right]^{2}\left[\left(\vec{\xi}_{\mu \mu+1} \cdot \vec{t}_{\mu \mu+1}\right) \vec{n}_{\mu \mu+1}-\left(\vec{\xi}_{\mu \mu+1} \cdot \vec{n}_{\mu \mu+1}\right) \vec{t}_{\mu \mu+1}\right] d \rho \\
& =\left[\vec{\xi}_{\mu \mu+1} \times \vec{l}\right] 72 \gamma_{\mu \mu+1}\left(\vec{n}_{\mu \mu+1}\right) \int_{-\infty}^{+\infty}\left[\frac{\partial \phi_{\mu+1}^{(0)}}{\partial \rho}\right]^{2} d \rho \\
& =\vec{\xi}_{\mu \mu+1} \times \vec{l}
\end{aligned}
$$

where we used the identity (36) in the last step. Hence the leading-order force balance at the multijunction is given as

$$
\vec{l} \times \sum_{\mu=0}^{m-1} \vec{\xi}_{\mu \mu+1}=\overrightarrow{0}
$$

We note that if we express the surface energies and the $\xi$ vectors in spherical polar coordinates such that the polar di- rection is parallel to $\vec{l}$, then from Eq. (2) the force balance at the multijunction (50) becomes

$$
\sum_{\mu=0}^{m-1} \gamma_{\mu \mu+1}(\phi, \theta) \vec{t}_{\mu \mu+1}-\frac{\partial g_{\mu \mu+1}(\phi, \theta)}{\partial \theta} \vec{n}_{\mu \mu+1}=\overrightarrow{0}
$$

We observe that the force balance contains the interaction of the $m$ surface tension terms $\gamma_{\mu \mu+1}(\phi, \theta) \vec{t}_{\mu \mu+1}$ acting tan- 


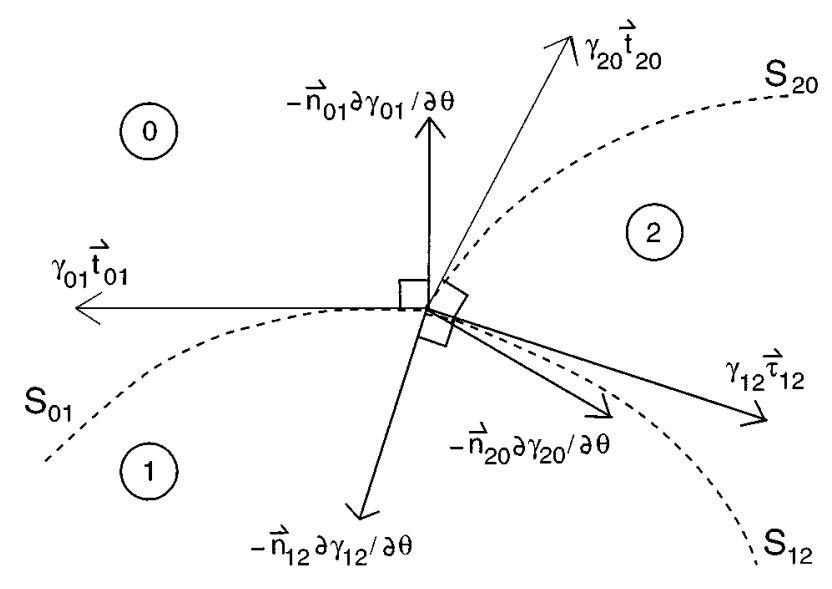

FIG. 3. Schematic diagram of the forces acting normally and tangentially to each interface at a trijunction of three phases labeled 0,1 , and 2 . The normal forces are the so-called Herring torque terms.

gentially to each interface. In addition, there are $m$ forces $\left[\partial \gamma_{\mu \mu+1}(\phi, \theta) / \partial \theta\right] \vec{n}_{\mu \mu+1}$ acting in the directions normal to each interface, as shown in Fig. 3 for the case of a trijunction $(m=3)$, which we identify as the so-called Herring torque terms. In the case of isotropic surface energies the Herring torque terms are zero and the conventional Young law is recovered.

\section{CONCLUSIONS}

In this paper we have developed a phase-field model pertinent to multiphase systems, such as grain boundaries and peritectic alloys, which includes anisotropy of both the surface energy and kinetic mobility of the interfaces. This model is a natural extension of an isotropic phase-field model originally proposed by Steinbach et al. [27]. A different phase field is used to represent each constituent bulk phase. The model has the advantage that the functional form of the anisotropy of both the surface energy and kinetic co- efficient of the interface between every pair of bulk phases can be specified independently by a suitable choice of the homogeneous degree-one functions $\gamma_{\alpha \beta}$ and $\mu_{\alpha \beta}$, respectively. The model does not seek to provide a realistic and detailed description of the internal structure of the interfaces and junctions, but rather provides a formal diffuse interface description that in the sharp interface limit provides the correct form of the Gibbs-Thomson-Herring equation at interfaces and the force balance at multijunctions. The model, in addition, provides a convenient setting in which to compute the motion of interfaces and junctions in systems involving more than two phases, although it has the disadvantage that the number of associated phase-field equations is proportional to the number of phases present, which correspondingly increases the computation requirements. We carefully extended the original isotropic model of Steinbach et al. so that we could exploit the recently generalized $\xi$-vector formalism developed by Wheeler and McFadden for diffuse interface models. This has allowed us to provide relatively short derivations of the sharp interface limits. It is worth noting that the divergence-free $\Xi$ tensor associated with time-independent solutions and the integral form of the associated conservation law (44) may be interpreted as a force balance that holds over any region that exists independently of the sharp interface limit. In fact, like the sharp interface conditions derived here at a multijunction, an alternative derivation of the sharp interface limit for an interface is possible directly from this force balance [33]. The conservation law $\vec{\nabla} \cdot \Xi=0$ itself is a consequence of the translational invariance of the underlying free-energy density of the system.

Finally, it is worth noting that we have also investigated the unsteady multijunction in the sharp interface limit and found that, to leading order, the static force balance is recovered. This is a consequence of the one-dimensional nature of a junction.

\section{ACKNOWLEDGMENT}

B.N. gratefully acknowledges the support provided by the German Research Foundation (DFG) under Grant No. SA $335 / 25-2$.
[1] J. S. Langer (private communication).

[2] G. Caginalp, Arch. Ration. Mech. Anal. 92, 205 (1986).

[3] J. B. Collins and H. Levine, Phys. Rev. B 31, 6119 (1985).

[4] O. Penrose and Paul C. Fife, Physica D 43, 44 (1990).

[5] S.-L. Wang, R. F. Sekerka, A. A. Wheeler, B. T. Murray, S. R. Coriell, R. J. Braun, and G. B. McFadden, Physica D 69, 189 (1993).

[6] A. A. Wheeler, W. J. Boettinger, and G. B. McFadden, Phys. Rev. A 45, 7424 (1992).

[7] G. Caginalp and W. Xie, Phys. Rev. E 48, 1897 (1993).

[8] J. A. Warren and W. J. Boettinger, Acta Metall. Mater. 43, 689 (1995).

[9] A. A. Wheeler, G. B. McFadden, and W. J. Boettinger, Proc. R. Soc. London, Ser. A 452, 495 (1996).

[10] A. Karma, Phys. Rev. E 39, 2245 (1994).
[11] A. A. Wheeler, W. J. Boettinger, and G. B. McFadden, Phys. Rev. E 47, 1893 (1993).

[12] N. A. Ahmad, A. A. Wheeler, W. J. Boettinger, and G. B. McFadden (unpublished).

[13] M. Conti, Phys. Rev. E 55, 701 (1997).

[14] M. Conti, Phys. Rev. E 55, 765 (1997).

[15] R. Kobayashi, Physica D 63, 410 (1993).

[16] G. Caginalp and P. C. Fife, Phys. Rev. B 33, 4940 (1986).

[17] G. B. McFadden, A. A. Wheeler, R. J. Braun, S. R. Coriell, and R. F. Sekerka, Phys. Rev. E 48, 2016 (1993).

[18] A. A. Wheeler and G. B. McFadden, Eur. J. Appl. Math. 7, 369 (1996).

[19] D. W. Hoffman and J. W. Cahn, Surf. Sci. 31, 368 (1972).

[20] J. W. Cahn and D. W. Hoffman, Acta Metall. 22, 1205 (1974).

[21] A. A. Wheeler and G. B. McFadden, Proc. R. Soc. London, Ser. A 453, 1611 (1997). 
[22] P. Sternberg, Rocky Mt. J. Math. 21, 799 (1991).

[23] Long-Qing Chen and Wei Young, Phys. Rev. B 50, 15752 (1994).

[24] Long-Qing Chen, Scr. Metall. Mater. 32, 115 (1995).

[25] S. Baldo, Ann. Inst. Henri Poincaré Phys. Theor. 7, 67 (1990).

[26] H. Garcke, B. Nestler, and B. Stoth, Physica D (to be published).

[27] I. Steinbach, F. Pezzolla, B. Nestler, J. Rezende, M. Seesselberg, and G. J. Schmitz, Physica D 94, 135 (1996).

[28] R. J. Braun, J. W. Cahn, G. B. McFadden, and A. A. Wheeler, Philos. Trans. R. Soc. London, Ser. A 355, 1787 (1997).
[29] M. Seesselberg, J. Tiaden, G. J. Schmitz, and I. Steinbach, in Proceedings of the Fourth Decennial International Conference on Solidification Processing, Sheffield, U.K., 1997, edited by J. Beech and H. Jones (BBR, Sheffield, 1997), p. 440.

[30] G. J. Schmitz, B. Nestler, and M. Seesselberg, Low Temp. Phys. 105, 1451 (1996).

[31] Herbert Goldstein, Classical Mechanics, 2nd ed. (AddisonWesley, Reading, MA, 1980).

[32] D. M. Anderson, G. B. McFadden, and A. A. Wheeler (unpublished).

[33] A. A. Wheeler (unpublished). 